\title{
Sleep laboratories reopening and COVID-19: a European perspective
}

\author{
Sophia Schiza ${ }^{1}$, Anita Simonds ${ }^{2}$, Winfried Randerath $\mathbb{0}^{3}$, Francesco Fanfulla ${ }^{4}$, \\ Dries Testelmans ${ }^{5}$, Ludger Grote $\mathbb{1}^{6}$, Joseph M. Montserrat ${ }^{7}$, \\ Jean-Louis Pepin ${ }^{8}$, Johan Verbraecken ${ }^{9}$, Refika Ersu ${ }^{10}$ and \\ Maria R. Bonsignore ${ }^{11}$
}

Affiliations: ${ }^{1}$ Sleep Disorders Center, Dept of Respiratory Medicine, Medical School, University of Crete, Heraklion, Greece. ${ }^{2}$ Sleep and Ventilation Unit, Royal Brompton and Harefield NHS Foundation Trust and National Heart and Lung Institute, London, UK. ${ }^{3}$ Institute of Pneumology at the University of Cologne, Bethanien Hospital, Solingen, Germany. ${ }^{4}$ Respiratory Function and Sleep Medicine Unit, IstitutiCliniciScientifici Maugeri IRCCS, Pavia, Italy. ${ }^{5}$ Clinical Dept of Respiratory Diseases, UZ Leuven - BREATHE, Department CHROMETA, KU Leuven, Leuven, Belgium. ${ }^{6}$ Sleep Disorders Centre, Respiratory Medicine, Sahlgrenska University Hospital and Centre for Sleep and Wake Disorders, Sahlgrenska Academy, Gothenburg University, Gothenburg, Sweden. ${ }^{7}$ Sleep Laboratory, Hospital Clinic, Universitat de Barcelona, Barcelona, and CIBER de Enfermedades Respiratorias, Madrid, Spain. ${ }^{8} \mathrm{HP} 2$ laboratory, INSERM U1042, University Grenoble Alpes and Grenoble Alpes University Hospital, Grenoble, France. ${ }^{9}$ Dept of Pulmonary Medicine and Multidisciplinary Sleep Disorders Centre, Antwerp University Hospital and University of Antwerp, Edegem (Antwerp), Belgium. ${ }^{10}$ Division of Pediatric Respirology, Children's Hospital of Eastern Ontario, University of Ottawa, Ottawa, ON, Canada. ${ }^{11}$ Sleep Disordered Breathing and Chronic Respiratory Failure Clinic, PROMISE Dept, University of Palermo, and IRIB, National Research Council (CNR), Palermo, Italy.

Correspondence: Maria R. Bonsignore, Sleep Disordered Breathing and Chronic Respiratory Failure Clinic, PROMISE Dept, University of Palermo, Via Liborio Guiffré, 13, 90127, Palermo, Italy. E-mail: marisa. bonsignoredirib.cnr.it

@ERSpublications

Specific precautions should be applied for starting activities in respiratory sleep centres during the COVID-19 epidemic https://bit.ly/3keRjii

Cite this article as: Schiza S, Simonds A, Randerath W, et al. Sleep laboratories reopening and COVID-19: a European perspective. Eur Respir J 2021; 57: 2002722 [https://doi.org/10.1183/13993003.02722-2020].

ABSTRACT Clinical activities regarding sleep disordered breathing (SDB) have been sharply interrupted during the initial phase of the coronavirus disease 2019 (COVID-19) epidemic throughout Europe. In the past months, activities have gradually restarted, according to epidemiological phase of COVID-19 and national recommendations. The recent increase in cases throughout Europe demands a reconsideration of management strategies of SDB accordingly. Diagnosis of SDB and initiation of treatment pose some specific problems to be addressed to preserve the safety of patients and health personnel. This perspective document by a group of European sleep experts aims to summarise some different approaches followed in Europe and United States, which reflect national recommendations according to the epidemiological phase of the COVID-19 infection. Respiratory sleep medicine is likely to change in the near future, and use of telemedicine will grow to avoid unnecessary risks and continue to provide optimal care to patients. In addition, the document covers paediatric sleep studies and indications for titration of noninvasive ventilation, as well as precautions to be followed by patients who are already on positive airway pressure treatment. A single consensus document developed by the European Respiratory Society and national societies would be desirable to harmonise SDB management throughout Europe.

This article has supplementary material available from erj.ersjournals.com

Received: 10 July 2020 | Accepted after revision: 3 Nov 2020

Copyright OERS 2021. This version is distributed under the terms of the Creative Commons Attribution NonCommercial Licence 4.0. 


\section{Introduction}

The coronavirus disease 2019 (COVID-19) pandemic has caused interruption of nonurgent health services in order to reduce the risk of infection and limit spread of the virus, especially in hospital environments. In addition, the staff of sleep laboratories have often been re-allocated to other medical services under pressure, for example respiratory medicine. This has caused the almost complete closure of sleep laboratories and clinics during lockdown in Europe [1] as in other parts of the world [2-4]. As a further precaution, children, pregnant women and patients at risk of severe forms of COVID-19 with pre-existing cardiorespiratory disease have been excluded from evaluation of sleep-related problems.

Several national guidance documents have been produced in Europe on the gradual reopening of sleep services, and differences between them reflect national policies, reimbursement rules or the relative role of mostly public versus private practices.

The aim of this perspective document is to propose a shared flexible model to be adapted locally, based on safe practice and the guidance and experiences developed in different European countries. This is particularly relevant since, after the initial phase of the pandemic, a new surge in the number of cases is occurring in several European countries. We analysed similarities and differences of some national recommendations regarding evaluation and treatment of obstructive sleep apnoea (OSA) in adults. Fewer recommendations were found on home noninvasive ventilation (NIV) and paediatric sleep disorders. Flowcharts approved by the European expert panel involved in this work provide the reader with graphical, easy-to-consult summaries for access to the sleep laboratory (COVID-19 screening; figure 1), diagnostic procedures (diagnosis; figure 2), and positive airway pressure (PAP) titration procedures (treatment; figure 3 ).

\section{National documents}

Boxes 1-3 and supplementary tables E1-E3 summarise the approaches suggested for screening for COVID-19, diagnosis, and treatment of sleep disordered breathing (SDB), respectively, by the main documents available to date [2-8]. The document developed by the American Academy of Sleep Medicine (AASM) was among the first to establish criteria for gradual reopening of sleep laboratories according to the local epidemiology of COVID-19 [2]. The approach followed by the Spanish Society of Pneumonology

No access in periods of high transmission in the community

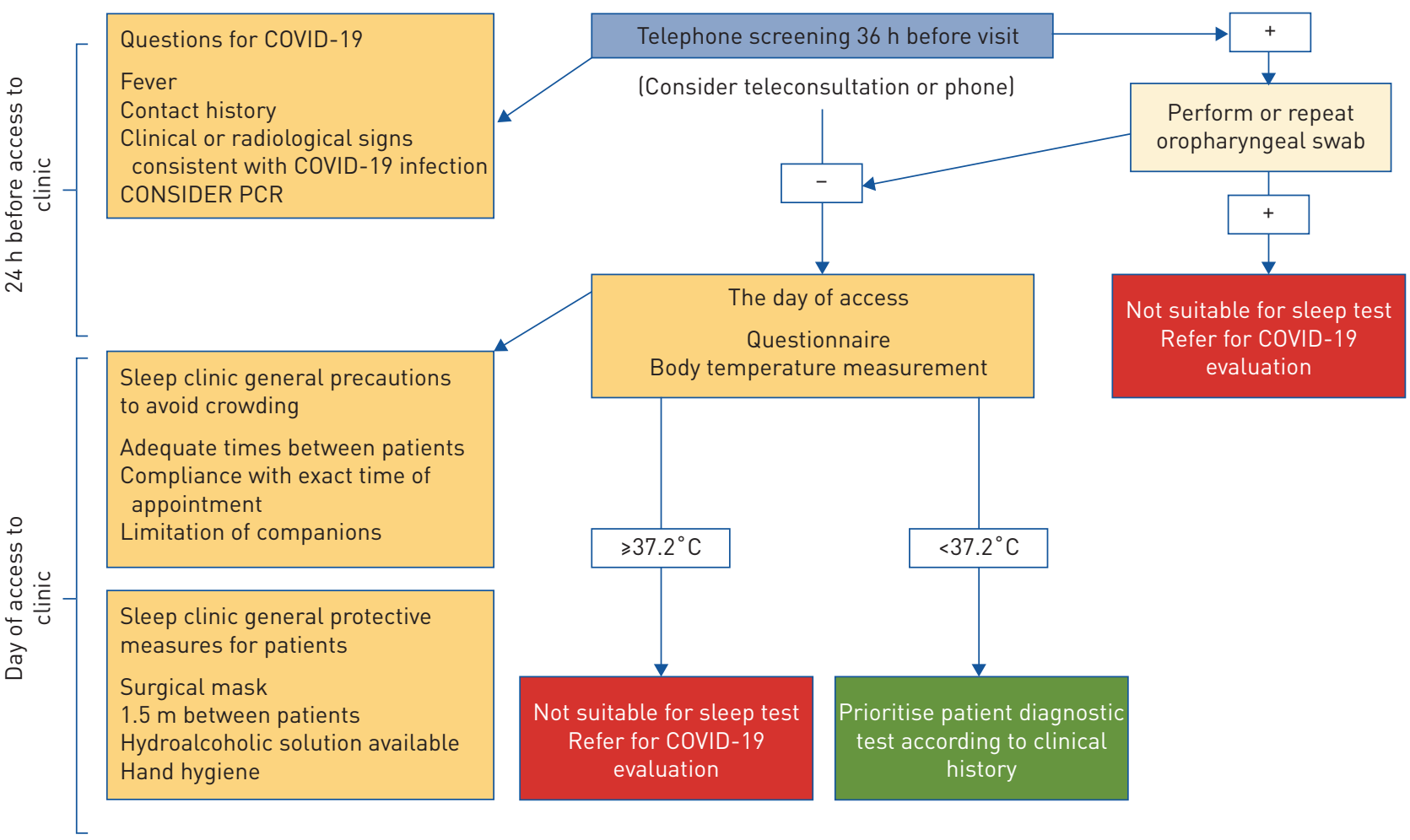

FIGURE 1 Flow chart summarising the screening procedures commonly adopted before access to the sleep laboratory or clinic. In phases of high coronavirus 2019 (COVID-19) transmission in the community, access to the sleep laboratory is limited to very urgent cases, and may require PCR testing. 


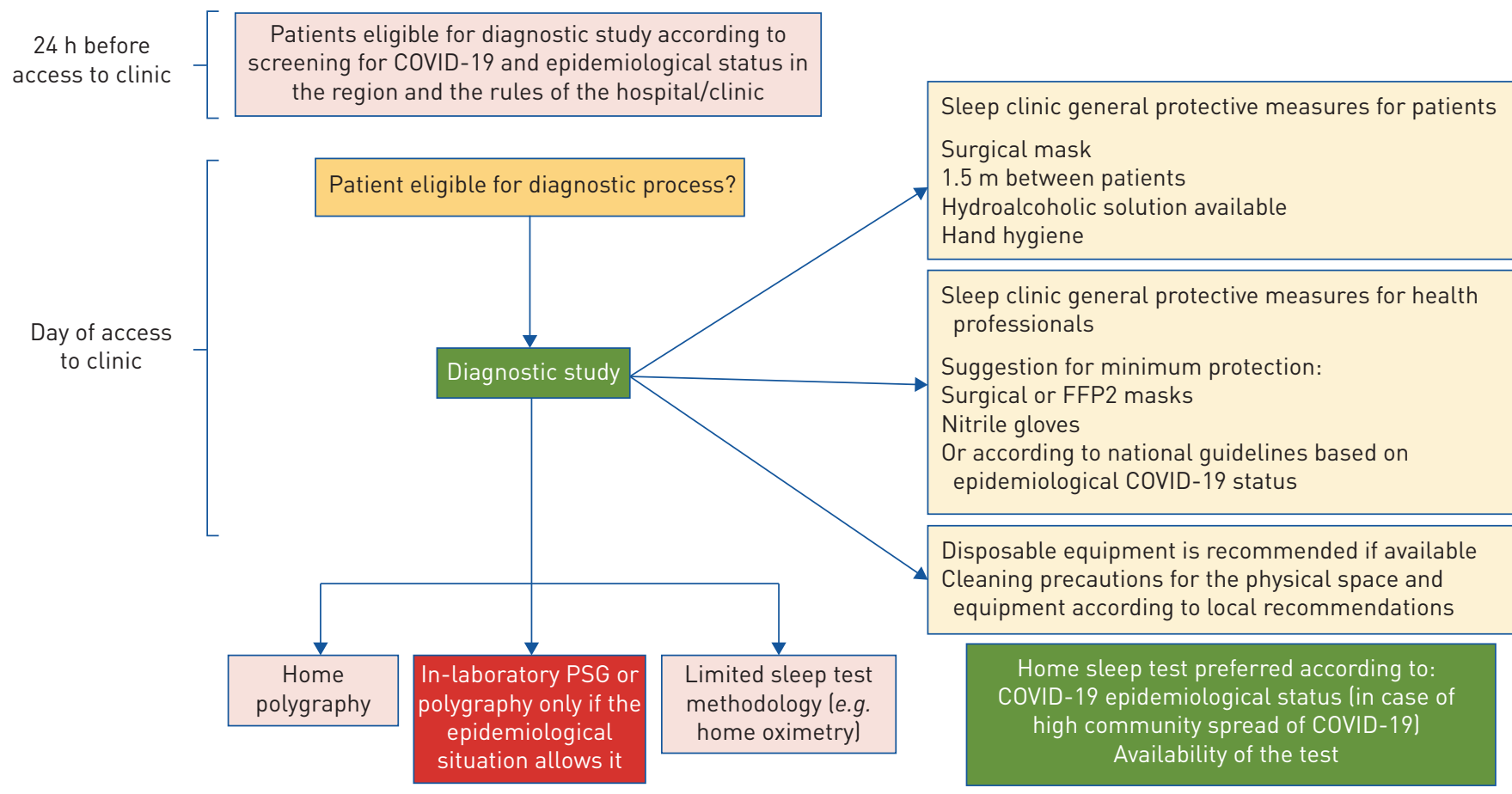

FIGURE 2 Flow chart summarising diagnostic procedures and precautions. In phases of high coronavirus 2019 (COVID-19) transmission in the community, PCR is usually required if in-laboratory polysomnography (PSG) is considered essential for diagnosis.

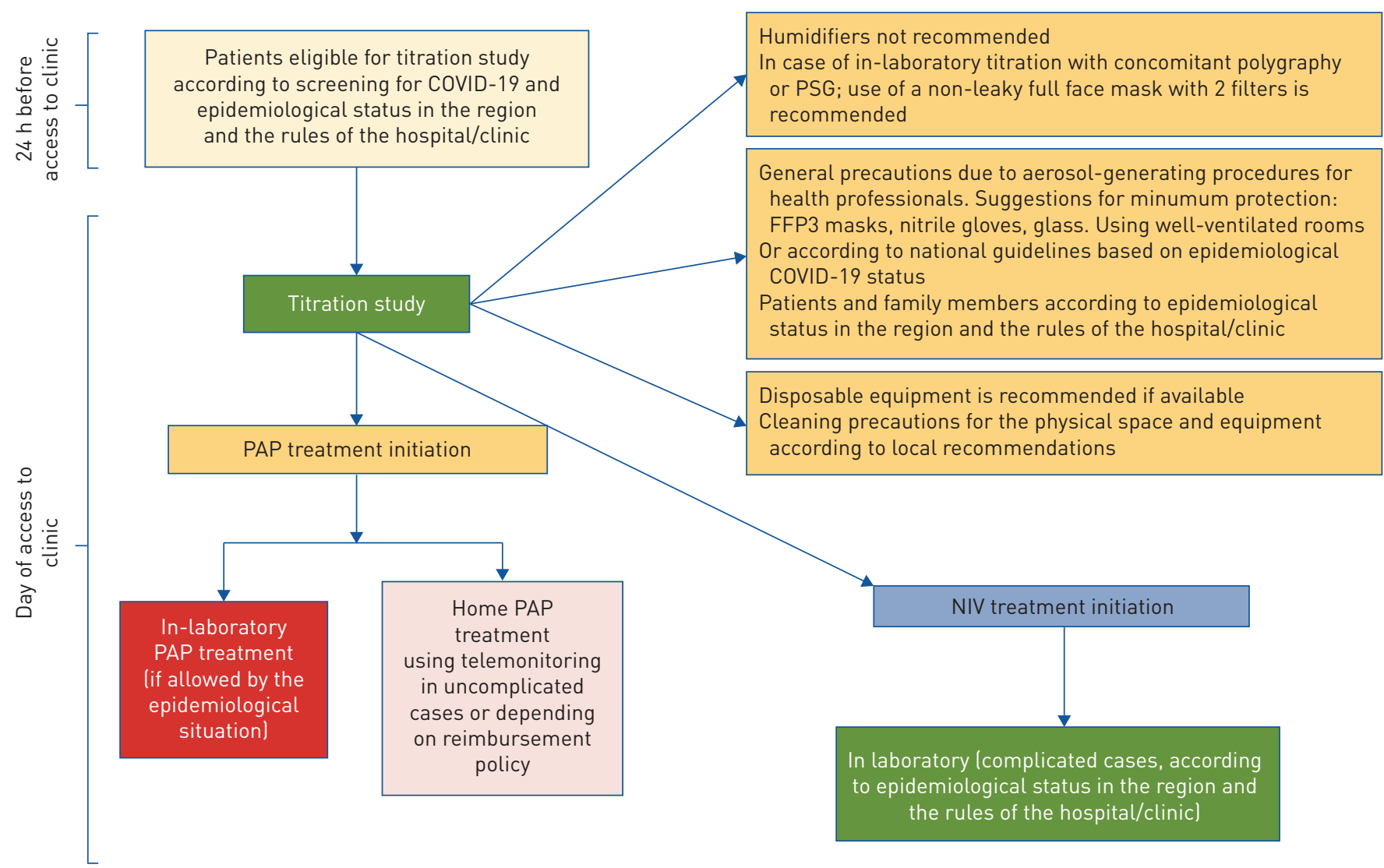

FIGURE 3 Flow chart summarising positive airway pressure (PAP) titration procedures and precautions. In phases of high coronavirus 2019 (COVID-19) transmission in the community, in-laboratory titration is avoided, and may require PCR testing. PSG: polysomnography; NIV: noninvasive ventilation. 
BOX 1 Screening for coronavirus 2019 (COVID-19): can the patient access the sleep clinic or the sleep laboratory?

In general, each sleep laboratory should take into account the local epidemiological status, the national mitigation strategies and adapt accordingly.

All guidelines agree on:

- If high incidence of COVID-19 in the community, no access to the sleep laboratory.

- Prioritisation of teleconsultation and/or telemedicine, with few (mainly urgent) face-to-face consultations.

- Assessment of patients prior to and at the time of the appointment for temperature, COVID-19 symptoms, contact history and testing status.

- General precautions to avoid crowding of patients in the waiting room.

- General protective measures for patients and staff (surgical or FFP2 masks, nitrile gloves).

Peculiarities:

- Patients $[3,4,6-8]$ and staff $[2,3,5,8]$ are screened by swab, based on COVID-19 epidemiological status and availability of the test.

- General precautions to avoid crowding of patients in the waiting room are stricter in UK [8] and USA [2, 3], based on available sleep laboratory facilities to minimise face-to-face contact, and guarantee sufficient air exchange in the room.

- In cases of high community spread of COVID-19, sleep laboratories may be closed for visits $[3,4]$.

and Thoracic Surgery (SEPAR) was similar, but used the lockdown phases according to the indications by the Spanish government reflecting the epidemiological situation [5]. In Italy, specific indications regarding sleep laboratories to be used all over the country have been published [6], as in Canada [4], Germany [7] and the UK [8]. More recently, a task force of the Association of Pulmonary, Critical Care, and Sleep Division Directors and the American Thoracic Society [3] took into consideration both the clinical needs of the patients and the epidemiological phase of COVID-19 pandemic. Each sleep clinic or laboratory will have to adapt its strategy to the current local prevalence of COVID-19 infection, with relatively loose precautions when the number of cases is low, and increasing levels of precautions when the number of cases and the risk of transmission are high.

BOX 2 Diagnosing patients at sleep laboratories during the coronavirus 2019 (COVID-19) pandemic

All guidelines agree on:

- Home sleep testing is preferred over in-laboratory testing in many countries.

- Limitation of in-laboratory polysomnography to urgent and high-risk patients in most countries.

- Assessment prior and at time of the in-person appointment for COVID-19 (symptoms, contact history or questionnaire).

- General precautions to avoid crowding of patients in the waiting room.

- General protective measures for patients and staff (surgical or FFP2 masks, nitrile gloves).

- Disposable probes, if available, in most countries.

- The sleep test device, sanitised after each use, should not be used for $\geqslant 72 \mathrm{~h}$ before use in another patient.

- Screening of patients and personal staff by swab in many countries, based on COVID-19 epidemiological status and availability of the test.

Peculiarities:

- For diagnostic study scheduling, age and comorbidities are taken into account in Spain [5], Italy [6] and USA $[2,3]$.

- Although in most countries home sleep testing is preferred, in Germany PSG remains the preferred diagnostic test [7]. In the UK [8] and Italy [6], limited sleep test methodology is used besides cardiorespiratory polygraphy.

- In the USA, the options of pickup/return of equipment by the patients, or a home delivery service, are suggested.

- The use of nasal cannulas is not recommended in Italy [6]; instead respiratory inductive plethysmography is suggested for the identification of respiratory events.

- General precautions to avoid crowding of patients in the waiting room are stricter in the UK [8] and USA $[2,3]$, based on available sleep laboratory facilities to minimise face-to-face contact and guarantee sufficient air exchange in the room.

- In cases of high community spread of COVID-19 [4, 6], in-laboratory PSG is usually postponed or PCR testing is required. 
BOX 3 Treating patients during the coronavirus 2019 (COVID-19) pandemic

All guidelines agree on:

- Home positive airway pressure and noninvasive ventilation treatment initiation using telemonitoring $[5,8]$.

- Humidifiers are usually not recommended during titration $[5,6,8]$.

- Disposable probes are recommended if available $[2,5,8]$.

- Use of a nonleaky full facemask with a filter added to the tubing is suggested in many countries.

- Equipment should be sanitised according to the manufacturer's instructions.

- Follow-up with telemedicine is preferred in most countries.

- General protective measures for patients and staff (FFP3 masks, nitrile gloves, glasses).

Peculiarities:

- In Germany, there are fewer restrictions for in-laboratory titrations and in-person visits [7]. In the USA and Italy (urgent cases), in-laboratory titrations are permitted if they take place in an airborne-infection isolation room $[2,6]$.

- In Spain [5] and the USA [2, 3], empirical treatment with delayed diagnostic testing could be considered in high-risk cases.

- In Spain [5], USA [2, 3] and Canada [4], PCR testing is required before the sleep study in the laboratory; negative PCR is required in Germany [7] before access to hospitals.

- In cases of high community spread of COVID-19, sleep laboratories are closed for visits.

\section{COVID-19 screening before access to the sleep clinic or laboratory}

All documents agree on the need to apply strict screening rules for COVID-19 before any in-person visits by patients to sleep centres (box 1, figure 1, supplementary table E1). These include a pre-triage phone-call the day before the planned appointment and prioritisation of tele-/video-consultation and/or telemedicine. Some national documents recommend avoidance of face-to-face visits according to the epidemiological situation, i.e. when contagion rate is increasing or high, and the clinical status of the patient allows successful management at home without risk. This strategy is more easily applicable to follow-up than to the first access visits. When there is high risk of COVID-19 transmission and the patient needs to be seen in person, COVID-19 testing before access to the sleep clinic is generally recommended.

\section{Face-to-face visits}

All documents agree on the need to apply strict screening rules for COVID-19 in case of in-person visits of the patient to the sleep centre (supplementary table E1). These include checking patients at the time of the appointment for temperature, COVID-19 symptoms and testing status upon their arrival at the sleep laboratory; only the patient is admitted to the visit room, unless an accompanying person is necessary; maintenance of safe distance and use of mask and gloves by the patients in some, but not all, countries; appropriate protective equipment worn by the health personnel; sanitisation of surfaces and cleaning of the room after the visit; and proper ventilation of the room between patients.

\section{Diagnostic tests}

In the acute epidemic phase, in-laboratory sleep tests should be avoided, and home sleep tests should be preferred (box 2, supplementary table E2, figure 2). Recommendations focus on the equipment to be sent to the patient's home, or taken by the patient, with detailed instructions on how to record a montage. Disposable equipment should be preferred, and external sanitisation of the devices is mandatory.

It has been hypothesised that, via the nasal cannula, the virus may reach the nasal pressure transducer, which cannot be sterilised. To avoid this risk, the AASM recommends no use of the polygraph for $72 \mathrm{~h}$ between tests on different patients [2]. This is a considerable limitation, especially considering the usual high demand for diagnostic sleep tests, possibly increased by the long interruption of sleep services. Low-cost ultraviolet C-machines have been proposed to sterilise nondisposable equipment [9] in alternative to standard hospital sanitisation procedures.

Single-use, disposable equipment is commercially available for sleep recordings, but the cost is higher than for traditional recordings. Other strategies to improve safety are based on substitution of nasal cannula/ thermistor with use of respiratory inductive plethysmography (RIP) [6].

Although portable sleep studies seem to be preferred by the majority of European centres, in-laboratory polysomnography (PSG) is obtained in some countries, e.g. Germany, Belgium, France. Negative results of the COVID-19 PCR test are usually, but not always, required before PSG.

Additional measures to reduce personnel exposure and minimise risk are decreasing the ratio of patients to technicians in the sleep laboratory during overnight studies and applying rotations of personnel. 
Titration studies

This is most challenging step, because of droplet/aerosol dispersion generated during PAP therapy, and the associated risk to the health personnel [10-12] (box 3, supplementary table E3). For OSA patients, use of automatic continuous positive airway pressure (CPAP) devices with remote monitoring is preferred, thus avoiding the return of the patient to the sleep laboratory. Ideally, the same PAP machine used for automatic titration should be prescribed to the patient, to avoid use of a single ventilator for titration in different patients. In some units a fixed pressure device is provided for titration, and adjustment of pressure is performed remotely by telemonitoring.

Droplet generation and dispersion, when vented masks are used, is not well documented, but might represent a real, preventable risk. A circuit using nonvented masks, two antibacterial/antiviral filters and a safety valve has been proposed, especially in cases of relevant epidemiological risk [13].

Common precautions are showing the patient how to put on the mask without turning the machine on, and using subsequent video consultation to support the patient in applying and using at home; no use of humidifier for in-laboratory titration; constant use of full or extended personal protective equipment (PPE) including FPP2/3 masks by health professionals; and in some European countries, i.e. in France, homecare providers can educate and start therapy during face-to-face visits at home. Principles for staff protection need to follow the same principles outlined for medical personnel.

A major problem is that to date, telemedicine services are reimbursed only in some European countries, strongly limiting their practical use. A survey on practice in several European sleep centres during the acute phase of the COVID-19 emergency showed that telemedicine was rarely used, while phone monitoring of patients during follow-up was quite frequent [1].

For NIV titration, the same precautions as with in-hospital CPAP titration would apply, including a COVID-19 PCR test before the procedure, according to national recommendations. Transcutaneous capnography should be obtained using disposable probes or transcutaneous measurement via ear clip. Healthcare personnel should use extended PPE during the titration [3-8]. An alternative approach is to show the patient how to use the ventilator as an outpatient, and then support initiation in the home with telemonitoring to facilitate titration. This is a modification of the home initiation protocol used by DUIVERMAN et al. [14].

For home ventilator setting titration, routine transcutaneous carbon dioxide $\left(\mathrm{CO}_{2}\right)$ monitoring is recommended if nonvented masks are used during the initial assessment. Home transcutaneous $\mathrm{CO}_{2}$ results can be transmitted by telemonitoring [14] or a transcutaneous $\mathrm{CO}_{2}$ monitor collected by the patient or couriered to the home with advice on measurement provided by video- or tele-consultation. The equipment is cleaned between patients using isopropyl alcohol cleaning wipes.

\section{Summary of precautions}

In summary, the expert panel suggests the following precautions:

Pre-visit screening (figure 1)

- Patients should be assessed $36 \mathrm{~h}$ prior to their in-person appointment and again at the time of the appointment before entering the facility. In case of fever, contact history and clinical or radiological signs consistent with COVID-19 infection, patients should be referred for diagnostic testing and screened again after a negative oropharyngeal swab.

In-person visits (figure 1)

- Visits on video or by phone calls should be preferred.

- Patients should be assessed again for COVID-19 status at the time of the appointment with questionnaires including fever, contact history and clinical or radiological signs consistent with COVID-19 infection and body temperature measurement.

- Febrile patients (temperature $\geqslant 37.2^{\circ} \mathrm{C}$ ) should be rescheduled and referred for COVID-19 evaluation.

- In case of normal temperature measurement $\left(<37.2^{\circ} \mathrm{C}\right)$ patients should be prioritised for diagnostic testing according to clinical history.

\section{Diagnostic tests (figure 2)}

- Home sleep testing is preferred over in-laboratory testing according to COVID-19 epidemiological status and availability of the test. 
- If patients are eligible for the diagnostic process, proper protective measures for patients and for all staff, as well as cleaning precautions for the physical space and equipment should be introduced.

- Ideally, patients should attend the sleep clinic alone.

- Disposable equipment is recommended if available.

- A negative nasopharyngeal swab is usually required for full in-laboratory PSG.

\section{Titration studies (figure 3)}

- Home PAP treatment initiation using telemonitoring is preferred over in-laboratory testing, in uncomplicated cases (depending on reimbursement policy).

- If patients are eligible for the titration study in the sleep laboratory, proper protective measures due to aerosol-generating procedures for patients and for all staff, as well as cleaning precautions for the physical space and equipment should be introduced.

- Humidifiers are not recommended.

- Use of a nonleaky full-face mask with two filters added to tubing is recommended.

- Home NIV treatment initiation using capnography is preferred over in-laboratory testing in stable cases.

What about patients already on PAP treatment?

The COVID-19 lockdown has caused disruption of sleep-activity rhythms in the population, with potential negative consequences on inflammatory and immune mechanisms $[15,16]$. The effects of the lockdown on patients already on PAP treatment are currently unknown. An interesting pro-con debate $[17,18]$ summarised the risks associated with interrupting or continuing home PAP/NIV treatment during the lockdown phase. However, according to a French study, adherence to PAP/NIV treatment increased during the lockdown, possibly due to staying at home and/or a higher perception of risk of developing respiratory disease [19]. In the current phase, it has been suggested that patients should continue home treatment [4].

The transmission of COVID-19 by the patient to his/her relatives at home during PAP treatment is a potential problem. Recommendations on this point are variable, given the sharing of common spaces at home by people in the family.

\section{Recommendations for patients on PAP treatment}

- In case of fever or respiratory infection, the patient should sleep in a separate room until resolution of symptoms or the results of PCR for COVID-19.

- Use of the humidifier is not indicated under such circumstances, since it may increase droplet and aerosol generation.

- If use of nonvented mask is preferred, an antibacterial/viral filter should be placed between the mask or tracheostomy and expiratory leak (or exhalation port). In some patients (adults or children) the added resistance from the filter can affect comfort. An exhalation valve may offer less resistance than a fixed leak. Close monitoring of arterial oxygen saturation and arterial $\mathrm{CO}_{2}$ tension is required, and the filter should be changed regularly. However, for patients (adults or children) using home ventilation, most units have not modified the mask or circuit used regularly.

To date, there are very few data on the clinical course of COVID-19 in OSA patients. Both OSA and obesity are pro-inflammatory conditions which may negatively interact with the COVID-19 infection [20, 21]. There are no data on the potential protective effect of OSA treatment in patients with COVID-19 infection.

Sleep studies in children during the COVID-19 pandemic

SDB in children encompasses obstructive sleep apnoea, central sleep apnoea and nocturnal hypoventilation, and is a very prevalent problem. As SDB has significant consequences including cardiovascular dysfunction and behavioural and neurocognitive sequelae, timely diagnosis and treatment is very important. The European Respiratory Society has published two guidelines for diagnosis and treatment of children with obstructive SDB [22, 23]. These guidelines recommend that children with symptoms of obstructive SDB need to be evaluated with objective testing such as polygraphy or PSG. Home sleep studies have not previously been routinely performed in children because of concerns about the adequacy of results [24].

In addition to obstructive SDB, children with certain underlying syndromes or underlying diseases may present with central sleep apnoea, which requires objective diagnostic testing. In addition, children with specific syndromes, such as Down syndrome or Prader-Willi syndrome, require surveillance for SDB, even 
in the absence of symptoms. KingshotT et al. [25] have shown a more encouraging outcome to cardiopulmonary polygraphy studies being performed at home in children, with $82 \%$ of clinical studies being successful at first attempt and parents confirming ease of use.

Sleep study resources are limited worldwide [26]. During the COVID-19 pandemic, most paediatric sleep laboratories have been closed and now there is an even bigger demand for sleep studies in children. Therefore, opening paediatric sleep laboratories in Europe is of utmost importance while keeping the sleep laboratories safe for the staff and children and their families. European countries have different approaches to infection control due to differences in prevalence rates and screening procedures in each country. General recommendations for safe practice in paediatric sleep laboratories in Europe follow.

\section{Recommendations for paediatric sleep studies}

- Sleep studies should be performed for paediatric patients whose care will be affected/delayed if a sleep study is not performed.

- Children should be accompanied by one caregiver during the sleep study.

- Prior to the sleep study, children and their caregivers should be screened for COVID-19 in accordance with local infection control practices. This should include, but is not limited to COVID-19 screening with a questionnaire. If the screening is positive, the sleep study should be deferred for 2 weeks and the child should be re-screened after 2 weeks.

- COVID-19 testing may be performed for children before the sleep study, based on community prevalence, available resources and local infection control recommendations.

- Sleep technicians may use PPE for droplet precautions during the sleep study. Different countries may have varying PPE protocols.

- If patients are started on a PAP therapy during the sleep study or if titration studies are planned, sleep technicians may use full extended PPE for droplet and aerosol precautions. Different countries may have varying PPE protocols for PAP therapy due to differences in prevalence rates.

- Home respiratory polygraphy and PAP initiation may be suitable in some selected cases.

\section{Discussion}

The COVID-19 pandemic has urged European health services to adjust procedures in order to increase safety. This is especially true and requires a cautious and flexible approach in the current phase of increasing COVID-19 cases throughout Europe. Use of telemedicine for SDB would be ideal for many patients, but needs to be implemented and reimbursed in many European countries before its use can become established at large. Our views have changed rapidly regarding use of telemedicine, since previous studies mainly addressed questions regarding early identification of problems or adherence to PAP treatment, whereas safety was not an issue [27]. Conversely, during the COVID-19 pandemic, telemedicine has been used to effectively provide patient care while limiting the risk of infection. A recent survey on how sleep medicine practices were affected by COVID-19 in the USA reported that the use of telemedicine and video consultation increased from $<25 \%$ to $>50 \%$ during the pandemic [28]. In addition, while in-laboratory testing decreased, use of home sleep apnoea testing was maintained by most laboratories [28]. Patient preferences indicated that patients rapidly adapted to the emerging needs associated with lockdown or limitations in mobility associated with the pandemic [28]. Use of telemedicine produced a faster change in practice in USA compared to Europe, possibly explained by more uniform regulations throughout USA, strong action by the AASM towards the implementation of telemedicine, and the larger flexibility of a private health system compared to the mostly public European health systems.

Diagnosis of SDB underwent some changes after the COVID-19 lockdown. A recent provocative article questioned the absolute need to perform PSG in all patients [29]. Home management of OSA has been shown to be cost-effective, with similar clinical results as those obtained by traditional management, especially in patients with high pre-test probability of OSA [30]. In addition, the use of home sleep tests may be a good alternative for paediatric sleep studies, and the field is moving its first steps towards this direction [31]. In the future, a fully developed "virtual sleep laboratory" may become a reality using video consultation and home sleep studies to diagnose SDB, titrate settings, solve problems when patients run into difficulties and assess of efficacy of treatment and adherence. Although a significant number of patients will require face-to-face contact, physical investigation of upper airway anatomy and dental status, and traditional sleep studies for optimal patient care and support, shifting towards a new paradigm of care would be especially vital at the time of COVID-19 pandemic. Since e-technology is already available, the challenge will be to put new management models into practice, with advantages for both patients and specialists, while maintaining a high level of care. Many studies have used telemedicine in OSA management, and showed its highest advantage at the start of CPAP treatment, when problems often arise 
and patients need additional support, with improved adherence to treatment $[32,33]$. However, we should go beyond the remote monitoring of treatment, to develop a new model of comprehensive care for patients on home PAP/NIV [34].

Alternative treatments for OSA should also be considered, such as oral appliances. SCHWARTz et al. [35] suggested that oral appliances should be first-line treatment during the pandemic, because of their safety. TANAKa and Almeida [36] have developed a set of precautions, including telemedicine, to optimise dental treatment for SDB in full safety.

The COVID-19 pandemic has profoundly changed the setting and attitudes of both patients and health professionals, and its foreseen duration for many months will provide the time for changes to become stable. A major problem is to guarantee access to care for all patients, and elderly or disadvantaged minorities may experience gaps in access to services and technology, and this is a problem to be addressed for the new setting to be effective. We need e-health services that are friendly, easy to use, cost effective and easy to access.

An interesting issue in the diagnosis of SDB is the development of safer sensors, for example nasal cannulas incorporating viral filters (not yet commercially available), or disposable diagnostic equipment, which may effectively increase safety of the patients, now and after the pandemic. Although RIP may help to diagnose respiratory events without using sensors on the patient's face, it is unlikely to replace traditional sensors for airflow, due to the relative instability of signals over time, and lack of sensitivity, especially in mild cases. Some solutions have been proposed, such as viral filters inserted in the nasal cannula circuit [37], or combination of RIP and tracheal sounds [38]. Finally, many smartphone apps may turn out to be useful for SDB diagnosis, but they are not yet validated.

For home PAP titration, it is advisable to use a PAP machine that can be assigned to the patient at prescription. Some laboratories have developed educational videos to show the patients the practical details of mask positioning and use of the PAP device at home, as well as troubleshooting of the most common problems encountered at the start of treatment.

When in-laboratory sleep study is absolutely necessary, as could be the case in both adults and children, a COVID-19 test is commonly requested, to protect both patient and staff. Table 1 reports the suggestions for patient and staff protection developed in the SEPAR document according to epidemiological phase [5].

TABLE 1 Summary of recommendations for management of patients with sleep disordered breathing according to epidemiological situation

\begin{tabular}{|c|c|c|c|}
\hline & Visits & Sleep test & $\begin{array}{l}\text { Evaluation for } \\
\text { COVID-19 }\end{array}$ \\
\hline $\begin{array}{l}\text { Stage 0: maximum } \\
\text { transmission rate }\end{array}$ & $\begin{array}{l}\text { Telemedicine } \\
\text { Phone }\end{array}$ & $\begin{array}{c}\text { No sleep studies, postpone initiation } \\
\text { of treatment } \\
\text { Home respiratory polygraphy in } \\
\text { high-risk cases }\end{array}$ & Clinical \\
\hline $\begin{array}{l}\text { Stage 1: improvement of } \\
\text { the pandemic situation }\end{array}$ & $\begin{array}{l}\text { Telemedicine } \\
\text { Phone }\end{array}$ & $\begin{array}{l}\text { Home respiratory polygraphy } \\
\text { (protection 1) } \\
\text { CPAP in high-risk patients: automatic } \\
\text { or telematic titration }\end{array}$ & Clinical \\
\hline $\begin{array}{l}\text { Stage 2: trend towards } \\
\text { normalisation of the } \\
\text { pandemic }\end{array}$ & $\begin{array}{l}\text { Telemedicine } \\
\text { Phone } \\
\text { In few cases: } \\
\text { face to face }\end{array}$ & $\begin{array}{l}\text { Home respiratory polygraphy } \\
\text { (protection 1) } \\
\text { PSG (protection 2) } \\
\text { CPAP: automatic or telematic } \\
\text { titration (protection 1); demonstration } \\
\text { in the laboratory (protection 2) }\end{array}$ & $\begin{array}{l}\text { PCR if PSG } \\
\text { Protection } 3 \text { if } \\
\text { titration in the } \\
\text { laboratory }\end{array}$ \\
\hline
\end{tabular}

COVID-19: coronavirus disease 2019; CPAP: continuous positive airway pressure; PSG: polysomnography. Protection 1: FFP2 mask, nitrile gloves, surgical gown (optional glasses or plastic shield); protection 2: PSG: the technician has to be comfortable while spending all the night with the patient; PCR is needed; FFP2 mask, surgical gown, hat, gloves and glasses (optional plastic face shield). Street clothes will not be used and the hospital uniform must be changed and cleaned daily; protection 3: FFP2/3 mask, nitrile gloves, surgical gown, hat, glasses/plastic face shields. Street clothes will never be used and the work uniform must be changed daily. It is recommended not to use open masks or circuits, but to use a closed circuit with an antiviral filter, turning on the equipment only when it is connected to the patient. Reproduced and modified from [5] with permission. 
Besides the oropharyngeal swab, rapid tests are becoming commercially available, and they could be a useful and rapid alternative to traditional PCR, and a major help, especially for paediatric patients.

In recent months, it has become clear that pandemic waves will recur, with different impact in different countries. Therefore, while local epidemiology must be the first variable to consider in adjusting management strategies, flexibility is necessary to adapt the general measures summarised in this article to the local setting, in conjunction with national recommendations. The COVID-19 pandemic represents a unique chance to revise our practice and to apply technology more effectively to improve the management of patients with SDB, while improving their safety. A single consensus document developed by the European Respiratory Society and national societies is desirable, to harmonise SDB management during pandemics throughout Europe.

Conflict of interest: None declared.

\section{References}

1 Grote L, McNicholas WT, Hedner J, et al. Sleep apnoea management in Europe during the COVID-19 pandemic: data from the European Sleep Apnoea Database (ESADA). Eur Respir J 2020; 55: 2001323.

2 American Academy of Sleep Medicine (AASM). COVID-19 Mitigation Strategies for Sleep Clinics and Sleep Centers-Reopening. August 27, 2020. www.aasm.org/covid-19-resources/covid-19-mitigation-strategies-sleepclinics-labs.

3 Wilson KC, Kaminsky DA, Michaud G, et al. Restoring pulmonary and sleep services as the COVID-19 pandemic lessens: from an Association of Pulmonary, Critical Care, and Sleep Division Directors and American Thoracic Society-coordinated task force. Ann Am Thorac Soc 2020; 17: 1343-1351.

4 Ayas NT, Fraser KL, Giannouli E, et al. Helping Canadian health care providers to optimize sleep disordered breathing management for their patients during the COVID-19 pandemic. Can J Respir Crit Care Sleep Med 2020; 4: 81-82.

5 Salord N, Montserrat JM, Javier Puertas F, et al. Unidades de sueño y consultas de ventilación mecánica no invasiva. Este apartado es un consenso entre la Sociedad Española de Neumología y Cirugía Torácica (SEPAR) y la Sociedad Española de Sueño (SES). [Sleep units and non-invasive mechanical ventilation consultations. This section is a consensus between the Spanish Society of Pulmonology and Thoracic Surgery (SEPAR) and the Spanish Sleep Society (SES)]. www.drive.google.com/file/d/1oORAlJ2lW6EPsOYvTbAYWJElQrpQP_dV/view.

6 Insalaco G, Dal Farra F, Braghiroli A, et al. Sleep breathing disorders in the COVID-19 era: Italian Thoracic Society organizational models for a correct approach to diagnosis and treatment. Respiration 2020; 99: 690-694.

7 Pfeifer M, Ewig S, Voshaar T, et al. Position paper for the state-of-the-art application of respiratory support in patients with COVID-19. Respiration 2020; 99: 521-542.

8 Association for Respiratory Technology and Physiology (ARTP) COVID Group. Guidelines for recommencing physiological services during the Coronavirus Disease 2019 (COVID-19) endemic phase. Date last updated: August 24, 2020. www.artp.org.uk/COVID19.

9 Kole AS. Home sleep testing in the era of COVID-19: a community perspective. J Clin Sleep Med 2020; 16: 1633.

10 Simonds AK, Hanak A, Chatwin M, et al. Evaluation of droplet dispersion during non-invasive ventilation, oxygen therapy, nebuliser treatment and chest physiotherapy in clinical practice: implications for management of pandemic influenza and other airborne infections. Health Technol Assess 2010; 14: 131-172.

11 Ferioli M, Cisternino C, Leo V, et al. Protecting healthcare workers from SARS-CoV-2 infection: practical indications. Eur Respir Rev 2020; 29: 200068.

12 Li J, Fink JB, Ehrmann S. High-flow nasal cannula for COVID-19 patients: low risk of bio-aerosol dispersion. Eur Respir J 2020; 55: 2000892.

13 Kryger M, Thomas R. Home PAP devices in patients infected with COVID-19. J Clin Sleep Med 2020; 16: 1217-1219.

14 Duiverman ML, Vonk JM, Bladder G, et al. Home initiation of chronic non-invasive ventilation in COPD patients with chronic hypercapnic respiratory failure: a randomised controlled trial. Thorax 2020; 75: 244-252.

15 Blume C, Schmidt MH, Cajochen C. Effects of COVID-19 lockdown on human sleep and rest-activity rhythms. Curr Biol 2020; 30: R795-R797.

16 Meira E Cruz M, Miyazawa M, Gozal D. Putative contributions of circadian clock and sleep in the context of SARS-CoV-2 infection. Eur Respir J 2020; 55: 2001023.

17 Barker J, Oyefeso O, Koeckerling D, et al. COVID-19: community CPAP and NIV should be stopped unless medically necessary to support life. Thorax 2020; 75: 367.

18 Baker JG, Sovani M. Case for continuing community NIV and CPAP during the COVID-19 epidemic. Thorax 2020; 75: 368.

19 Attias D, Pepin JL, Pathak A. Impact of COVID-19 lockdown on adherence to continuous positive airway pressure (CPAP) by obstructive sleep apnoea patients. Eur Respir J 2020; 56: 2001607.

20 McSharry D, Malhotra A. Potential influences of obstructive sleep apnea and obesity on COVID-19 severity. J Clin Sleep Med 2020; 16: 1645.

21 Tufik S, Gozal D, Antunes Ishikura I, et al. Does obstructive sleep apnea lead to increased risk of COVID-19 infection and severity? J Clin Sleep Med 2020; 16: 1425-1426.

22 Kaditis AG, Alonso Alvarez ML, Boudewyns A, et al. ERS statement on obstructive sleep disordered breathing in 1- to 23-month-old children. Eur RespirJ 2017; 50: 1700985.

23 Kaditis AG, Alonso Alvarez ML, Boudewyns A, et al. Obstructive sleep disordered breathing in 2- to 18-year old children: diagnosis and treatment. Eur Respir J 2016; 47: 69-94. 
24 Kirk V, Baughn J, D'Andrea L, et al. American Academy of Sleep Medicine position paper for the use of a home sleep apnea test for the diagnosis of OSA in children. J Clin Sleep Med 2017; 13: 1199-1203.

25 Kingshott RN, Gahleitner F, Elphick HE, et al. Cardiorespiratory sleep studies at home: experience in research and clinical cohorts. Arch Dis Child 2019; 104: 476-481.

26 Katz SL, Witmans M, Barrowman N, et al. Paediatric sleep resources in Canada: the scope of the problem. Paediatr Child Health 2014; 19: 367-372.

27 Bruyneel M. Telemedicine in the diagnosis and treatment of sleep apnoea. Eur Respir Rev 2019; 28: 180093.

28 Johnson KG, Sullivan SS, Nti A, et al. The impact of the COVID-19 pandemic on sleep medicine practices. J Clin Sleep Med 2020. In press doi:10.5664/jcsm.8830.

29 Patel SR, Donovan LM. The COVID-19 pandemic presents an opportunity to reassess the value of polysomnography. Am J Respir Crit Care Med 2020; 202: 309-310.

30 Masa JF, Corral J, Sanchez de Cos J, et al. Effectiveness of three sleep apnea management alternatives. Sleep 2013; 36: 1799-1807.

31 Ross KR, Redline S. Is it time to head home for the night? Home sleep testing in young children. Ann Am Thorac Soc 2020; 17: 1207-1209.

32 Hwang D, Chang JW, Benjafield AV, et al. Effect of telemedicine education and telemonitoring on continuous positive airway pressure adherence. The Tele-OSA randomized trial. Am J Respir Crit Care Med 2018; 197: $117-126$.

33 Schoch OD, Baty F, Boesch M, et al. Telemedicine for continuous positive airway pressure in sleep apnea. A randomized, controlled study. Ann Am Thorac Soc 2019; 16: 1550-1557.

34 Suarez-Giron M, Bonsignore MR, Montserrat JM. New organisation for follow-up and assessment of treatment efficacy in sleep apnoea. Eur Respir Rev 2019; 28: 190059.

35 Schwartz D, Addy N, Levine M, et al. Oral appliance therapy should be prescribed as a first-line therapy for OSA during the COVID-19 pandemic. J Dent Sleep Med 2020; 7.

36 Tanaka Y, Almeida FR. What can a dentist and dental sleep apnea researcher do under COVID-19 lockdown? J Clin Sleep Med 2020; 16: 1641-1643.

37 Perger E, Trentin R, Lombardi C, et al. Safe sleep apnea tests during Covid-19 pandemic: a new practical proposal. Sleep Med 2020; 75: 341-342.

38 Sabil A, Marien C, LeVaillant M, et al. Diagnosis of sleep apnea without sensors on the patient's face. J Clin Sleep Med 2020; 16: 1161-1169. 